PROCEEDINGS OF THE

AMERICAN MATHEMATICAL SOCIETY

Volume 125, Number 5, May 1997, Pages 1517-1522

S 0002-9939(97)03756-8

\title{
A NOTE ON THE FUNDAMENTAL GROUPS OF MANIFOLDS WITH ALMOST NONNEGATIVE CURVATURE
}

\author{
GABJIN YUN
}

(Communicated by Christopher Croke)

\begin{abstract}
We show that given $n$ and $D, v>0$, there exists a positive number $\epsilon=\epsilon(n, D, v)>0$ such that if a closed $n$-manifold $M$ satisfies $\operatorname{Ric}(M) \geq$ $-\epsilon, \operatorname{diam}(M) \leq D$ and $\operatorname{vol}(M) \geq v$, then $\pi_{1}(M)$ is almost abelian.
\end{abstract}

\section{INTROODUCTION}

In this note, we give some properties of the fundamental groups of Riemannian manifolds of almost nonnegative curvature. In [12], G. Wei proved that there exists an $\epsilon=\epsilon(n, D, v)>0$ such that the fundamental group $\pi_{1}(M)$ of a Rienannian $n$-manifold $M$ with $\epsilon$-almost nonnegative Ricci curvature, diameter $\operatorname{diam}(M) \leq D$ and volume $\operatorname{vol}(M) \geq v$ has polynomial growth of order $\leq n$.

Recall that by a theorem of Gromov a finitely generated group is of polynomial growth if and only if it is almost nilpotent, i.e., it contains a nilpotent subgroup of finite index $([9])$. So, together with this, $\pi_{1}(M)$ is almost nilpotent in Wei's theorem.

On the other hand, note that for any nilmanifold $N^{n}$ which is not a torus, $\pi_{1}(N)$ has polynomial growth of order $>n([10],[13])$. Thus we might expect a little stronger conclusion of Wei's theorem.

To extend the well-known results on manifolds of nonnegative Ricci curvature to manifolds of almost nonnegative Ricci curvature, a major problem is the splitting conjecture by Fukaya and Yamaguchi ([7], [15]). Recently, Cheeger and Colding ([3]) have announced that they have solved this conjecture; see also [5] for other results on manifolds with almost nonnegative Ricci curvature.

Theorem 1 (Splitting Theorem). Let $(X, p)$ be the pointed Hausdorff limit of a sequence $\left(M_{i}, p_{i}\right)$ of complete Riemannian $n$-manifolds with Ric $\left(M_{i}\right)>-\epsilon_{i} \rightarrow 0$. Then the splitting theorem holds for $X$, i.e., if $X$ contains a line, then $X$ splits $\mathbf{R} \times X^{\prime}$ isometrically.

Using this theorem together with other results, we can prove the following theorem.

Received by the editors April 11, 1995 and, in revised form, November 29, 1995.

1991 Mathematics Subject Classification. Primary 53C20; Secondary 57S20.

Key words and phrases. Almost non-negative curvature, almost nilpotent and abelian group.

(C)1997 American Mathematical Society 
Theorem 2 (Main Theorem). Given $n$ and $D, v>0$, there exists a positive number $\epsilon=\epsilon(n, D, v)$ such that if a closed n-manifold $M$ satisfies

$$
\operatorname{Ric}(M) \geq-\epsilon, \quad \operatorname{diam}(M) \leq D, \quad \operatorname{vol}(M) \geq v,
$$

then $\pi_{1}(M)$ is almost abelian, i.e., $\pi_{1}(M)$ contains an abelian subgroup of finite index.

The examples of manifolds discussed above show that this theorem is not true without the assumption of a lower volume bound. However in low dimensions we will show that the lower volume bound can be removed; See theorem 5 .

\section{Preliminaries}

In [8], Gromov introduced the Hausdorff distance between metric spaces. See [6], [7], [8], [15] for results about Hausdorff distance.

Now let us describe a result which is connected to the splitting property and isometry group. Let $Y$ be a compact metric space, and $G$ a closed subgroup of the group of isometries of the product $\mathbf{R}^{k} \times Y$. Since $G$ preserves the splitting $\mathbf{R}^{k} \times Y$, the projection $\phi: G \rightarrow \operatorname{Isom}\left(\mathbf{R}^{k}\right)$ is well defined. In fact, we have the following lemma.

Lemma 1.1 ([7]). For each $\delta>0$, there exists a normal subgroup $G_{\delta}$ of $G$ such that

(1) $G / G_{\delta}$ is discrete, and

(2) there exists an exact sequence

$$
1 \rightarrow G_{\delta} \rightarrow G \rightarrow \Lambda \rightarrow 1
$$

where $\Lambda=G / G_{\delta}$ contains a finite index free abelian subgroup of rank not greater than $\operatorname{dim}\left(\mathbf{R}^{k} / \phi(G)\right)$.

Proof. Let us indicate how to construct the normal subgroup $G_{\delta}$. For a complete proof, see [7].

Let $K$ be the kernel of $\phi$. Then $K$ acts on $Y$. Set

$$
K_{\delta}=\langle\{\gamma \in K \mid d(\gamma(x), x)<\delta, \quad \text { for all } \quad x \in Y\}\rangle,
$$

where $\langle\{\}\rangle$ denotes the group generated by the set \{\} . Then $K_{\delta}$ is a normal subgroup of $G$ and the natural projection $\pi: G \rightarrow G / K_{\delta}$ is well-defined. Moreover, one can show that $G / K_{\delta}$ is a Lie group.

We now define

$$
G_{\delta}=\pi^{-1}\left(\left(G / K_{\delta}\right)_{o}\right),
$$

where $\left(G / K_{\delta}\right)_{o}$ denotes the identity component of $G / K_{\delta}$.

Remark 1. Note in particular that if $G_{\delta}=\{1\}$, then $G$ is almost abelian.

Lemma 1.2 ([13]). If $\Gamma$ is a finitely generated group, and $\Lambda$ a sugroup of $\Gamma$ of finite index, then $\Lambda$ is also finitely generated.

A solvable group $\Gamma$ is called polycyclic if there is a normal series

$$
\Gamma=\Gamma_{0} \supset \Gamma_{1} \supset \cdots \supset \Gamma_{k}=\{1\}
$$

with every quotient $\Gamma_{i} / \Gamma_{i+1}$ finite or infinite cyclic. 
Lemma 1.3. Any finitely generated group $\Gamma$ of polynomial growth contains a torsion free nilpotent subgroup of finite index.

Proof. By a theorem of Gromov ([9]) we have that $\Gamma$ is almost nilpotent. That is, there is a nilpotent subgroup $\Lambda$ of finite index. Further, by Lemma 1.2 this implies that $\Lambda$ is finitely generated. Now it follows from [11] that every subgroup of $\Lambda$ is finitely generated. Thus, by [13], $\Lambda$ is polycyclic and therefore contains a torsion free subgroup of finite index.

Finally, we recall two theorems of Anderson ([1]). These theorems of Anderson play an important role in the proof of the main theorem.

Theorem $3([1])$. Given $k$ and $D, v>0$, in the class of compact n-dimensional Riemannian manifolds $M$ such that

$$
\operatorname{Ric}(M) \geq-(n-1) k^{2}, \quad \operatorname{vol}(M) \geq v, \quad \operatorname{diam}(M) \leq D,
$$

there are only finitely many isomorphism classes of $\pi_{1}(M)$.

Theorem 4 ([1]). Let $M$ be a compact $n$-manifold satisfying the bounds $\left(^{*}\right)$. If $\gamma$ is a curve in $M$ with $[\gamma]^{p} \neq 0$ in $\pi_{1}(M)$ for all $p \leq v_{k}(2 D) / v$, then the length of $\gamma$ satisfies

$$
l(\gamma) \geq \frac{D v}{v_{k}(2 D)}
$$

Here $v_{k}(r)$ denotes the volume of a geodesic ball of radius $r$ in the space form of constant curvature $-k$.

\section{Proof of the Main Theorem}

We are now in position to prove the main theorem.

Proof of the Main Theorem. By Wei's theorem [12], there is a positive number $\epsilon_{o}=$ $\epsilon_{o}(n, D, v)$ such that if a closed $n$-manifold $M$ satisfies $\operatorname{Ric}(M) \geq-\epsilon_{o}, \operatorname{vol}(M) \geq v$ and $\operatorname{diam}(M) \leq D$, then $\pi_{1}(M)$ is a finitely generated group of polynomial growth of order $\leq n$.

Suppose the theorem is not true. Then there is a sequence of closed manifolds $M_{i}$ with

$$
\operatorname{Ric}\left(M_{i}\right) \geq-\epsilon_{i} \rightarrow 0, \quad \epsilon_{i}<\epsilon_{o}, \quad \operatorname{vol}\left(M_{i}\right) \geq v, \quad \operatorname{diam}\left(M_{i}\right) \leq D,
$$

and $\pi_{1}\left(M_{i}\right)$ is not almost abelian.

By lemma 1.3, there is a torsion free nilpotent subgroup $\Gamma_{i}$ of $\pi_{1}\left(M_{i}\right)$ of finite index. Note that $\Gamma_{i}$ cannot be almost abelian. Moreover, by theorem 3 above, we may assume that

$$
\text { the index }\left[\pi_{1}\left(M_{i}\right): \Gamma_{i}\right] \text { is uniformly bounded }
$$

After possibly passing to a subsequence, we may by Gromov's compactness theorem ([8]) assume that $M_{i}$ converges to a compact length space $X$. Since $X$ might not a manifold, we need to consider the action of $\Gamma_{i}$ on the universal cover $\widetilde{M}_{i}$. For $p_{i}$ in $\widetilde{M}_{i}$, consider $\left(\widetilde{M}_{i}, \Gamma_{i}, p_{i}\right)$.

From an extension of Gromov's compactness theorem due to Fukaya and Yamaguchi (see [7] and [15]) we have that there exist a length space $(Y, q)$ and a closed subgroup $G$ of $\operatorname{Isom}(Y)$ such that $\left(\widetilde{M}_{i}, \Gamma_{i}, p_{i}\right)$ subconverges to a triple $(Y, G, q)$ with respect to the pointed equivariant Hausdorff distance. 
The splitting theorem together with the assumption that $\operatorname{diam}\left(M_{i}\right) \leq D$ implies that $Y$ is isometric to a product $\mathbf{R}^{k} \times Y_{o}$, where $Y_{o}$ is compact.

On the other hand, by theorem 4 above, there exists a positive number $\delta=$ $\delta\left(n, D, v, \epsilon_{o}\right)>0$ such that $\Gamma_{i}(\delta)=\left\{\gamma \in \Gamma_{i}: d\left(p_{i}, \gamma\left(p_{i}\right)\right)<\delta\right\}=\{1\}$. This implies that $G(\delta / 2)=\{\gamma \in G: d(q, \gamma(q))<\delta / 2\}=\{1\}$, and so by the costruction of $G_{\delta}$ the corresponding normal subgroup $G_{\delta / 2}$ in lemma 1.1 is trivial. Therefore, by lemma 1.1, $G / G_{\delta / 2}=G$ is discrete and $G$ contains a finite index free abelian subgroup of rank $\leq k$. Moreover by $\left.{ }^{* *}\right) \mathbf{R}^{k} \times Y_{o} / G$ is compact. Thus by theorem 3.10 in [7], $\Gamma_{i}$ is isomorphic to $G$ for $i$ sufficiently large. This contradicts the fact that $\Gamma_{i}$ is not almost abelian.

Next we will prove the same property of the fundamental groups of almost nonnegative sectional curvature without a lower volume bound. In [7], Fukaya and Yamaguchi proved that there exists a positive small number $\epsilon$, depending only on the dimension, such that the fundamental groups of manifolds satisfying $K_{M} \cdot \operatorname{diam}(M)^{2} \geq-\epsilon$ must be almost nilpotent. If we bound the growth of the fundamental group by dimension, we get the same result as theorem 2 for the low dimension.

Theorem 5. Let $n \leq 4$. There exists a positive number $\epsilon=\epsilon(n)$ such that if $M$ is a closed Riemannian n-manifold satisfying

(1) $K_{M} \cdot \operatorname{diam}(M)^{2} \geq-\epsilon$,

(2) $\pi_{1}(M)$ has polynomial growth of order $\leq n$, then $\pi_{1}(M)$ is almost abelian.

Proof. If $n \leq 3$, then this is trivial. In fact, by the Fukaya-Yamaguchi theorem and Lemma $1.3, \pi_{1}(M)$ contains a finitely generated torsion free nilpotent subgroup $\Gamma$ of finite index. In particular $\operatorname{growth}(\Gamma) \leq n \leq 3$. So, if $\Gamma$ is not abelian, it contains a Heisenberg group as a subgroup, and it is known that the growth of the Heisenberg group is exactly $4([10])$. Therefore $\Gamma$ must be abelian, i.e., $\pi_{1}(M)$ is almost abelian.

Now consider the case $\operatorname{dim}(M)=n=4$. We may assume that $\pi_{1}(M)$ is an infinite group. From the fibration theorem of Yamaguchi ([15]) we obtain that

$$
F \rightarrow M^{*} \rightarrow S^{1}
$$

is a fibration with fiber $F$. Moreover, we have an exact sequence

$$
1 \rightarrow \pi_{1}(F) \rightarrow \pi_{1}\left(M^{*}\right) \rightarrow \mathbf{Z} \rightarrow 1 .
$$

In particular,

$$
\pi_{1}\left(M^{*}\right) / \pi_{1}(F) \cong \mathbf{Z} .
$$

Since growth $\left(\pi_{1}\left(M^{*}\right)\right)=\operatorname{growth}\left(\pi_{1}(M)\right) \leq n=4$, Gromov's splitting theorem for growth of groups $([9])$ implies that

$$
\operatorname{growth}\left(\pi_{1}(F)\right)=\operatorname{growth}\left(\pi_{1}\left(M^{*}\right)\right)-1 \leq 3=\operatorname{dim}(F) .
$$

Therefore, $\pi_{1}(F)$ is almost abelian since $F$ admits a metric (the induced metric) of $\epsilon$-nonnegative curvature as above.

On the other hand, from a theorem of Fukaya and Yamaguchi ([7], [15]), there exists a finite cover $F^{*}$ of $F$ such that

(1) $F^{*} \sim S^{3} \quad$ (homotopic),

(2) $F^{*} \cong S^{1} \times S^{2} \quad$ (diffeomorphic), 
(3) $F^{*} \cong T^{3} \quad$ (diffeomorphic).

Note that a non-toral nilmanifold is excluded because the fundamental group of $F$ is almost abelian.

We will show that $\pi_{1}(M)$ is almost abelian in each case. In the first case, we have $\pi_{1}\left(F^{*}\right)=0$ and so $\pi_{1}(F)$ is finite. Thus from $(\dagger)$, we see that $\pi_{1}\left(M^{*}\right)$ is almost abelain and so is $\pi_{1}(M)$.

In the second case, we have $\pi_{1}\left(F^{*}\right)=\mathbf{Z} \oplus \Lambda$, where $\Lambda$ is a finite group. Thus (†) becomes

$$
1 \rightarrow \mathbf{Z} \oplus \Lambda \rightarrow \pi_{1}\left(M^{*}\right) \rightarrow \mathbf{Z} \rightarrow 1 .
$$

It is easy to show that $\pi_{1}\left(M^{*}\right)$ has a torsion free abelian subgroup which is isomorphic to $\mathbf{Z} \oplus \mathbf{Z}$ of finite index, and so is $\pi_{1}(M)$.

For the last case, we have $b_{1}(F)=b_{1}\left(F^{*}\right)=3$ (here $b_{1}$ is the first Betti number), and so $F \cong T^{3}$ by a theorem of Yamaguchi ([14], [15]). Thus we have

$$
T^{3} \rightarrow M^{*} \rightarrow S^{1}
$$

and

$$
1 \rightarrow \mathbf{Z}^{3} \rightarrow \pi_{1}\left(M^{*}\right) \rightarrow \mathbf{Z} \rightarrow 1,
$$

and therefore $\operatorname{growth}\left(\pi_{1}\left(M^{*}\right)\right)=4$.

Let $\Gamma$ be a torsion free subgroup of $\pi_{1}\left(M^{*}\right)$ of finite index. We claim that $\Gamma$ is abelian. Note that $\operatorname{growth}(\Gamma)=4$. So if $\Gamma$ is not abelian, we see that $\Gamma$ is the Heisenberg group $H$. However $H=\Gamma$ does not contain $\mathbf{Z}^{3}$ as a subgroup, and $\mathbf{Z}^{3} \subset \pi_{1}\left(M^{*}\right)$. Since the index of $\Gamma$ in $\pi_{1}\left(M^{*}\right)$ is finite, this is impossible.

Remark 2. If $n \geq 5$, then the theorem is clearly false. For example, take a leftinvariant metric $g_{\epsilon}$ on a nilpotent Lie group $N$ of dimension $\geq 3$, with sectional curvature $K_{\left(N, g_{\epsilon}\right)} \geq-\epsilon$, and $g_{o}$ the standard metric on the 2-sphere $S^{2}$. Consider the product $M=N \times S^{2}$ with product metric $g=g_{\epsilon}+g_{o}$. Then $(M, g)$ satisfies the conditions in the theorem above, but the fundamental group of $M$ is not almost abelian. I believe however that for $n \geq 5$ such a splitting is the only possibility. Namely, if $M$ satisfies the above conditions, then it seems likely that either $\pi_{1}(M)$ is almost abelian or $M$ is a bundle with nilmanifold fiber over a compact space $S$ with finite fundamental group.

\section{ACKNOWLEDGEMENT}

The author would like to thank his advisor Michael T. Anderson for many suggestions and helping him complete this note. He is also grateful to T. Colding for pointing out several important things.

\section{REFERENCES}

1. M. T. Anderson, Short geodesics and gravitational instantons, J. Diff. Geometry 31 (1990), 265-275. MR 91b:53040

2. $\quad$ On the topology of complete manifolds of nonnegative Ricci curvature, Topology 29 (1990), 41-55. MR 91b:53041

3. J. Cheeger and T. Colding, preprint.

4. J. Cheeger and D. Gromoll, The splitting theorem for manifolds of nonnegative Ricci curvature, J. Diff. Geometry 6 (1971), 119-128. MR 46:2597

5. T. Colding, Ricci curvature and volume convergence, preprint.

6. K. Fukaya, Theory of convergence for Riemannian orbifolds, Iapan J. Math. 12 (1986), 121160. MR 89d:53083 
7. K. Fukaya and T. Yamaguchi, The fundamental groups of almost nonnegatively curved manifolds, Ann. Math. 136 (1992), 253-333. MR 93h:53041

8. M. Gromov, (rédige par J. Lafontaine et P. Pansu), Structure métrique pour les variété riemanniennes, Cedic/Fernand Nathan, Paris, 1982. MR 85e:53051

9. Groups of polynomial growth and expanding maps, Inst. Hautes Études Sci. Publ. Math. No. 53 (1981), 53-73. MR 83b:53041

10. J. Milnor, A note on curvature and fundamental group, J. Diff Geometry 2 (1968), 1-7. MR 38:636

11. M. S. Raghunathan, Discrete subgroups of Lie groups, Springer-Verlag, Berlin, 1972. MR $\mathbf{5 8 : 2 2 3 9 4 a}$

12. G. Wei, On the fundamental groups of manifolds with almost nonnegative Ricci curvature, Proc. Amer. Math. Soc 110 (1990), 197-199. MR 90m:53058

13. J. Wolf, Growth of finitely generated solvable groups and curvature of Riemannian manifolds, J. Diff. Geometry 2 (1968), 421-446. MR 40:1939

14. T. Yamaguchi, Collapsing and pinching under a lower curvature bound, Ann. of Math. 133 (1991), 317-357. MR 92b:53067

15. , Manifolds of almost nonnegative curvature, MPI, 1993.

Department of Mathematics, SUNy at Stony Brook, Stony Brook, New York 11794

Current address: Department of Mathematics and GARC, Seoul National University, Seoul, Korea 151-742

E-mail address: gabjin@math.snu.ac.kr 